Epidemiology and Infection

cambridge.org/hyg

\section{Original Paper}

*Those who contributed equally to the work.

Cite this article: Singh C, Naik BN, Pandey $S$, Biswas B, Pati BK, Verma M, Singh PK (2021). Effectiveness of COVID-19 vaccine in preventing infection and disease severity: a case-control study from an Eastern State of India. Epidemiology and Infection 149, e224, 1-9. https://doi.org/10.1017/ S0950268821002247

Received: 9 August 2021

Revised: 5 October 2021

Accepted: 5 October 2021

Key words:

Case-control studies; COVID-19; effectiveness; India; SARS-CoV-2; vaccines

Author for correspondence:

Bijit Biswas,

E-mail: drbijitbiswas@gmail.com (c) The Author(s), 2021. Published by Cambridge University Press. This is an Open Access article, distributed under the terms of the Creative Commons Attribution licence (http://creativecommons.org/licenses/by/4.0/), which permits unrestricted re-use, distribution and reproduction, provided the original article is properly cited.

\section{CAMBRIDGE} UNIVERSITY PRESS

\title{
Effectiveness of COVID-19 vaccine in preventing infection and disease severity: a case-control study from an Eastern State of India
}

\section{Chandramani Singh ${ }^{1, \star}\left(\mathbb{D}\right.$, Bijaya Nanda Naik ${ }^{1, \star}\left(\mathbb{D}\right.$, Sanjay Pandey ${ }^{1, \star}(\mathbb{D})$ Bijit Biswas $^{1, \star}\left(\mathbb{D}\right.$, Binod Kumar Pati ${ }^{2}$, Manisha Verma ${ }^{1}$ and Prabhat Kumar Singh ${ }^{3}$}

${ }^{1}$ Department of Community and Family Medicine, All India Institute of Medical Sciences, Patna, Bihar, India; ${ }^{2}$ Department of Microbiology, All India Institute of Medical Sciences, Patna, Bihar, India and ${ }^{3}$ Department of Anaesthesiology and Critical Care, All India Institute of Medical Sciences, Patna, Bihar, India

\begin{abstract}
Effectiveness of corona virus disease-19 (COVID-19) vaccines used in India is unexplored and need to be substantiated. The present case-control study was planned to elicit the effectiveness of COVID-19 vaccines in preventing infection and disease severity in the general population of Bihar, India. This case-control study was conducted among people aged $\geq 45$ years during April to June 2021. The cases were the COVID-19 patients admitted or visited All India Institute of Medical Sciences (AIIMS), Patna, Bihar, India, and were contacted directly. The controls were the individuals tested negative for severe acute respiratory syndrome coronavirus-2 (SARS CoV-2) at the Virology laboratory, AIIMS-Patna and contacted telephonically for collection of relevant information. The vaccine effectiveness (VE) was calculated by using the formula ( $\mathrm{VE}=1$ - odds ratio). The adjusted VE for partial and full vaccination were estimated to be $52.0 \%$ (95\% confidence interval (CI) 39.0-63.0\%) and $83.0 \%$ (95\% CI 73.0-89.0\%) respectively for preventing SARS CoV-2 infection. The sub-group analyses of the cases have shown that the length of hospital stays (LOS) (partially vaccinated: 9 days vs. unvaccinated: 12 days; $P=0.028$ ) and the severity of the disease (fully vaccinated: $30.3 \%$ vs. partially vaccinated: $51.3 \%$ and unvaccinated: $54.1 \% ; P=0.035$ ) were significantly low among vaccinated compared to unvaccinated individuals. To conclude, four out of every five fully vaccinated individuals are estimated to be protected from contracting SARS CoV-2 infection. Vaccination lowered LOS and chances of development of severe disease.
\end{abstract}

\section{Research in context:}

\section{Evidence before this study}

We searched PubMed using the following search terms for observational studies ('ChAdOx1 nCoV-19' or 'Oxford' or 'AstraZeneca' or 'BBV152') and ('COVID-19' or 'SARS-CoV-2') and ('vaccine' or 'vaccination') and 'effectiveness' with no language or year restriction on 23 July 2021. We retrieved VIVALDI cohort study from the UK which reported that protection offered by a single-dose Oxford-AstraZeneca vaccine from SARS-CoV-2 infection to be $68 \%$ (at 35-48 days) among adults aged $\geq 65$ years. Another cohort study from north-west London documented $74 \%$ risk reduction of testing positive for SARS-CoV-2 after 28 days of receiving first dose of ChAdOx1 nCoV-19. Public Health England test-negative case-control study observed vaccine effectiveness (VE) in terms of COVID-19-related symptom prevention of first dose of Oxford-AstraZeneca vaccine to be $60 \%$ between 28 and 34 days which increased to $73 \%$ after 35 days among older adults aged $\geq 70$ years.

\section{Added value of this study}

Through adaptation of case-control design, we investigated the combined effectiveness of in use Covishield (Oxford-AstraZeneca) and Covaxin (Bharat Biotech) SARS-CoV-2 vaccines of India. Unadjusted combined VE was found to be $45.0 \%(95 \% \mathrm{Cl} 30.0-56.0)$ in the partially vaccinated group and $77.0 \%(95 \% \mathrm{Cl} 65.0-84.0 \%)$ in the fully vaccinated group in preventing SARS-CoV-2 infection. After adjusting with potential confounders, the VE for partial and full vaccination were estimated to be $52.0 \%(95 \% \mathrm{Cl} 39.0-63.0 \%)$ and $83.0 \%(95 \% \mathrm{Cl} 73.0-$ $89.0 \%)$, respectively. We also adjusted VE with COVID-appropriate behaviour as per World Health Organisation (WHO) recommendations which other prior studies ignored. Vaccination was also found to confer protection against experiencing a severe disease and lowered hospital stay.

\section{Implications of all the available evidence}

This is one of the earlier evidences on real-world VE of COVID-19 vaccines used in the world's largest COVID-19 vaccination drive. Addition of the evidences generated in the present study with prior pieces of evidences available in this context will help in restoring faith regarding SARS-CoV-2 vaccination among the beneficiaries of it. This will also consolidate trust of policymakers on vaccination who issued emergency approval to these vaccines on public interest amid pandemic.

\section{Introduction}

Vaccination is one of the safest and cost-effective public health interventions for infectious disease prevention and control, especially in a pandemic situation [1]. The corona virus disease-19 (COVID-19) pandemic which has affected 31174322 individuals and has claimed 
414482 lives in India as of 21 July 2021 is not an exception [2]. In the absence of a curative therapy, and the difficulty of imposing strict COVID-appropriate behaviours, the demand for safe and effective vaccine emerged early, leading to its development at an unprecedented pace. As of 15 April 2021, two COVID-19 vaccines, Covaxin and Covishield, are in use in India. The Covaxin is a virion-inactivated severe acute respiratory syndrome coronavirus-2 (SARS-CoV-2) vaccine developed and produced by Bharat Biotech whereas the Covishield is a recombinant chimpanzee adenovirus vector vaccine manufactured by Serum Institute of India (SII) with technology transfer from the Oxford University and AstraZeneca. The Covaxin, administered with two doses 4 weeks apart, is reported to have overall efficacy of $77.8 \%$, and $93.4 \%$ against the severe symptomatic disease. The Covaxin is also reported to have good efficacy (65.2\%) against the Delta (B.1.617.2) variant of SARS-CoV-2 [3]. The Covishield vaccine, also administered with two doses, claimed to have an efficacy of $55.1 \%$ for a shorter time interval ( $\leq 6$ weeks) between the two doses. The increase in interval between the two doses of the Covishield vaccine to 12 weeks or more was claimed to confer $147 \%$ higher protection (vaccine efficacy: $81 \cdot 3 \%$ ) compared to the short interval [4].

India started the world's largest vaccination drive with the use of the Covaxin and the Covishield vaccine both in a phased manner against COVID-19 on 16 January 2021 [5]. In the initial two phases, only the healthcare workers (HCWs) and the front-line workers irrespective of their age were eligible for vaccination. The COVID vaccines were made available for the general public of the country in a phased manner starting from 1 March 2021 (for the elderly and the individuals aged above 45 years with the comorbidities) and from 1 May 2021 (for all adults) [6]. The interval between the doses of the Covaxin is 4 weeks and the Covishield is revised from 4 weeks at the start of the vaccination drive to 12-16 weeks as of May 2021 [7].

Both the COVID-19 vaccines in India demonstrated good clinical efficacy and received the approval from the Drug Controller of India (DGCI) for emergency use. However, the real-world effectiveness of these vaccines have been largely unexplored and not documented in Indian settings till date [3, 8-10]. Moreover, the vaccine effectiveness (VE) assessment is required to overcome the vaccine hesitancy, restoration of faith and increase the acceptance of COVID-19 vaccines among the target population to prevent and control the current pandemic [11]. As of 21 April 2021, only $8.3 \%$ and $1.3 \%$ of the total population of the country could be vaccinated with first and second doses of SARS-CoV-2 jab, respectively [12]. The case-control design is the cost-effective and rapid way of the VE assessment during an epidemic situation [10]. Previously case-control study designs have been used in the post-licencing effectiveness of the oral cholera vaccine, rota virus vaccine and the influenza vaccines [13-15]. The case-control design has already been adopted by studies from other countries for the COVID-19 VE assessment [16-18]. In this context, the present case-control study was planned to estimate the VE of COVID-19 vaccines in preventing infection and disease severity in an Eastern State of India.

\section{Materials and methods}

We conducted an unmatched case-control study at All India Institute of Medical Sciences (AIIMS), situated in the ancient city of Patna beside the holy river Ganges between April and June 2021. All consecutive SARS CoV-2-positive patients who have availed services from our institute and the individuals who were tested negative for SARS CoV-2 at our Virology laboratory during the study period were the potential cases and controls for the study, respectively. We considered only the first visit of the cases for enrolment. Notably, none of our recruited cases were reinfected during the study period which was one of the preset exclusion criteria. In either group, only one participant from a family having multiple eligible participants (index-one) was considered. The controls with a history of COVID-19 or influenzalike illness (ILI) in the preceding 3 months from the day of data collection were excluded. The Institutional Ethical Committee (IEC) of AIIMS, Patna (Ref. AIIMS/Pat/IEC/2020/ 706) approved the study protocol.

The odds ratio (OR) of contracting infection following COVID-19 vaccination was calculated to be 0.3 with the anticipated VE of 70\%. During April 2021, the first dose COVID-19 vaccination coverage among the eligible general population of Bihar was found to be $3.7 \%$. Considering the vaccination coverage (3.7\%) and OR (0.3), the ratio of controls to cases $2: 1,95 \%$ precision and $80 \%$ power, the minimum sample size for the study was calculated to be 507 cases and 1013 controls using the online software OpenEpi $[12,19,20]$. A total of 577 cases and 1154 controls were selected during the study period and considered for the final analyses. The post-hoc power analysis using OpenEpi software showed the adequacy of final sample size in our study (full vaccination (cases vs. control: $5.7 \%$ vs. $17.4 \%$; power: $100.0 \%$ ); partial vaccination (cases vs. control: $26.7 \%$ vs. $34.3 \%$; power: $88.8 \%)$ ) [21].

We approached consecutive COVID-19 patients aged $\geq 45$ years (as during protocol development vaccination for 18-44 had not started) with a valid report (rapid antigen $(\mathrm{Ag})$ or reverse transcriptase polymerase chain reaction (RT-PCR) for SARS-CoV-2) who have attained COVID dedicated flu clinic of our institute during the study period. Consented individuals were included as cases and were interviewed face-to-face. The information for severe and moribund cases was obtained from accompanying family members or friends directly. The highest disease severity of hospitalised cases was later retrieved from their medical records, while for home isolated ones, it was inquired telephonically. Line lists of RT-PCR-negative individuals aged $\geq 45$ years were collected on a daily basis from our Virology laboratory during the study period. We telephonically approached individuals from these line lists at random within 3 days of their report and the consenting individuals were included as controls. All interviews were done by trained resident doctors of our institute. Details of case and control recruitment process are depicted in Fig. 1.

The study schedule was largely adopted from the World Health Organization (WHO) guidelines on the evaluation of COVID-19 effectiveness (2021) and modified in the local context [10]. The final study schedule comprised of age, sex, occupation, chronic co-morbidity status, history of $(\mathrm{H} / \mathrm{O})$ hospitalisation for chronic co-morbidities in past 5 years, COVID-19 vaccination profile (vaccination status; name of the vaccine, number of doses, date of first dose, date of second dose; the predominant reason for not getting vaccinated), symptom profile, H/O ILI, past COVID-19 infection and high-risk contact with COVID-19 case or suspect since March 2020, COVID-appropriate behaviour profile of last 14 days (use of mask while going outdoors, adherence to social distancing, avoidance of crowded places, handwashing before touching face); date of RT-PCR/rapid Ag test; hospitalisation status and disease characteristics (for cases only) (disease 


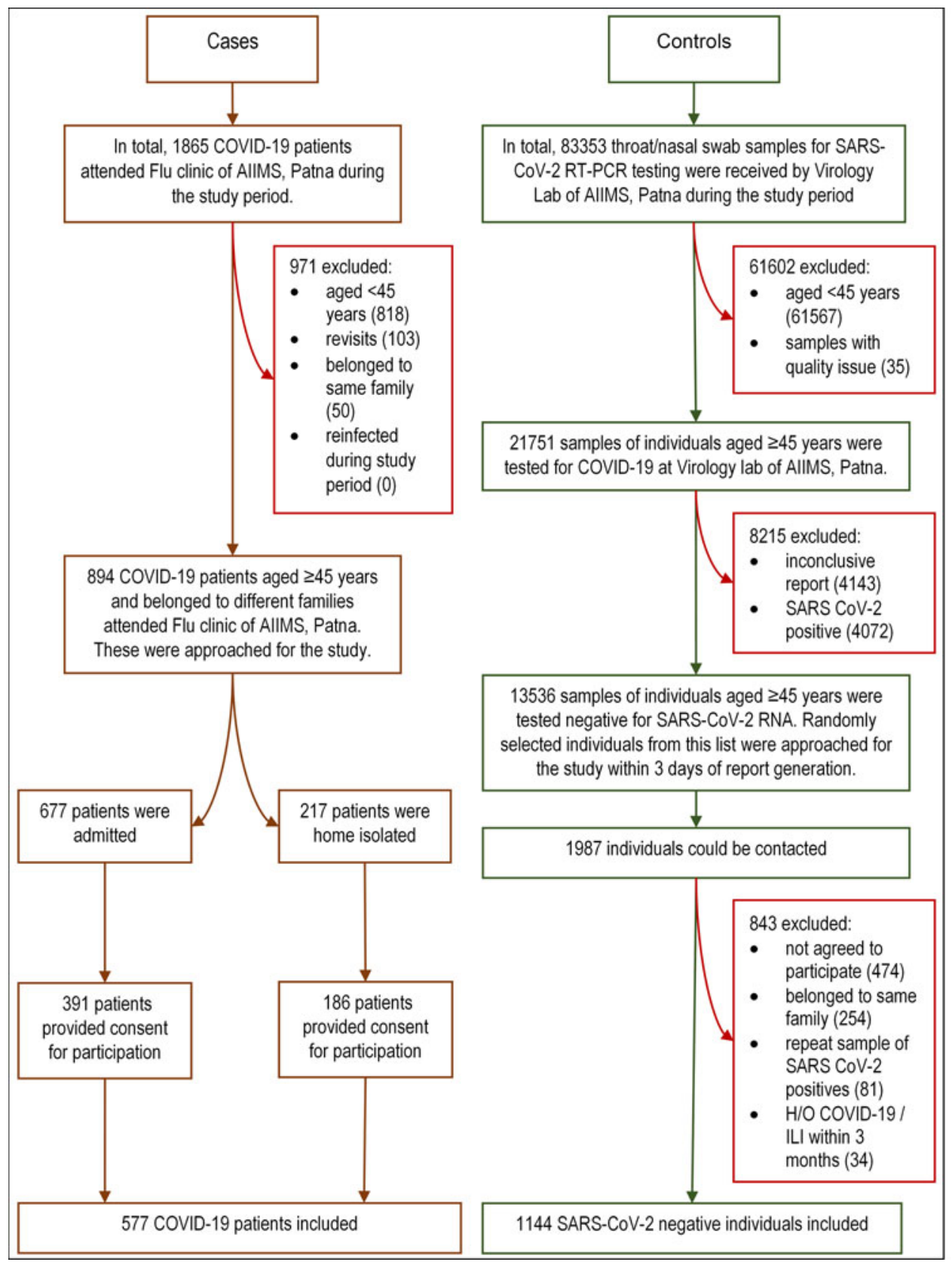

Fig. 1. Flowchart showing recruitment of the cases and controls. COVID-19, corona virus disease-19; RT-PCR, reverse transcriptase polymerase chain reaction; SARS-CoV-2, severe acute respiratory syndrome coronavirus-2; RNA, ribonucleic acid; H/O, history of; ILI: influenza-like illness.

severity, length of hospital stay (LOS) and final treatment outcome).

\section{Operational definitions}

Severity of COVID-19, Mild: No complaints of breathlessness and normal pulse oxygen saturation $\left(\mathrm{SpO}_{2}\right): \geq 94 \%$ and respiratory rate (RR): $<24$ breaths per minute (bpm); Moderate: Complaint of breathlessness and/or SpO2: 90-93\% on room air and/or RR: 24$30 \mathrm{bpm}$ with no features of severe disease (i.e. shock, organ dysfunction); Severe: SpO2: $<90 \%$ on room air and/or RR: $>30 \mathrm{bpm}$ and/or features of severe disease (i.e. shock, organ dysfunction) $[22,23]$. Four items of the COVID-appropriate behaviour were scored as follows: always (0), sometimes (1) and never (2). Scores of these four items were summed-up to obtain the total score where a higher score indicated more COVID-inappropriate behaviour. The definitions used for ascertaining COVID-19 vaccination status were as follows, unvaccinated: At the time of testing for SARS-CoV-2, one has not received jab or was within 14 days of first jab; partially vaccinated: did the testing for SARS-CoV-2, $\geq 14$ days of first jab or before 14 days of second jab; fully vaccinated: did the testing for SARS-CoV-2, $\geq 14$ days after second jab.

\section{Statistical analysis}

The information collected was entered using Epicollect5 (an open-source data collection software) [24]. The data entered were downloaded and imported to statistical package for social 
sciences (SPSS) (version 22.0) for analysis. The quantitative variables were expressed as median (interquartile range (IQR)). The categorical variables were expressed as proportion and percentages. The Mann-Whitney $U$ test and Kruskal-Wallis test were used to compare medians across binomial and ordinal variables, respectively. Bonferroni's test was used for post-hoc analysis of ordinal variables. Pearson's $\chi^{2}$ test was used to test the association between categorical variables. The $\chi^{2}$ adjusted standardised test was used for higher order contingency table $(>2 \times 2)$ to identify significant pair. The protective effect of COVID vaccines for contracting COVID-19 infection was statistically tested using univariable logistic regression followed by multivariable logistic regression, and quantification was expressed as OR. We adjusted for all actual or potential confounders (age, sex, occupation, COVID-inappropriate behaviour score, chronic co-morbidity, H/O hospitalisation, ILI, prior COVID-19 and high-risk contact with a case or suspect) in the multivariable logistic regression model. Unadjusted and adjusted VE were calculated using the following formulae: $(\mathrm{VE}=(1-\mathrm{OR}) \times 100 \%)$. We used $95 \%$ confidence level for all the statistical tests used.

\section{Result}

The median age of the cases was higher than the controls while male gender constituted about two-third of the study population in both the groups. About three-fifth (58.8\%) of the cases and one-fourth of the controls (28.8\%) were having at least one chronic co-morbidity. About two-fifth of the cases (42.1\%) and three-fifth of the controls $(61.4 \%)$ have received at least a single jab. About two-third and one-fifth of the study participants in both the groups have received Covishield and Covaxin, respectively (Table 1).

Inaccessibility to vaccination centres (overall: $31.8 \%$; cases: $26.3 \%$, controls: $35.9 \%$ ) was reported to be the predominant reason for not getting vaccinated followed by fear of side effects (overall: 21.8\%; cases: $25.7 \%$, controls: $18.9 \%$ ) and sceptical about VE (overall: 13.9\%; case: 15.6\%, controls: 12.6\%) (Fig. 2).

Unadjusted VE was found to be $45.0 \%$ (95\% CI 30.0-56.0) in the partially vaccinated group and $77.0 \%$ (95\% CI 65.0\%-84.0\%) in the fully vaccinated group in preventing SARS-CoV-2 infection. After adjusting with potential confounders like age, sex, occupation, COVID-inappropriate behaviour score, chronic co-morbidity, H/O hospitalisation for chronic co-morbidity, ILI, past COVID-19 infection and high-risk contact with a COVID-19 case or suspect, the VE for partial and full vaccination were estimated to be $52.0 \%$ (95\% CI 39.0-63.0\%) and $83.0 \%(95 \%$ CI 73.0-89.0\%), respectively. A full vaccination course was calculated to have provided $31.0 \%$ additional protection compared to partial vaccination in preventing SARS-CoV-2 infection (Table 2).

The difference in median LOS among the cases between partially vaccinated (9 days (IQR 5-13 days)) and unvaccinated (12 days (IQR 6-16 days)) was statistically significant $(P=0.028)$. The fully vaccinated COVID patients had a lower probability of having the severe disease on arrival (18.2\%) compared to the partially vaccinated $(31.8 \%)$ and the unvaccinated $(42.6 \%)$ cases $(P=$ 0.022). Overall, the fully vaccinated COVID patients were also found to have less likelihood of experiencing severe disease (30.3\%) during their illness compared to partially vaccinated $(51.3 \%)$ and unvaccinated $(54.1 \%)$ cases $(P=0.035)$. The final outcome (death/recovery) was statistically indifferent across the different vaccination groups (Table 3 ).
VE and disease characteristics of Covaxin and Covishield subgroups are presented in Supplementary Tables S1-S4.

\section{Discussion}

The Government of India is rolling out the emergency use of two COVID vaccines in an unprecedented manner to control the ongoing pandemic, which is now open to all adults of the country. Still, the coverage of vaccination is less despite 6 months into the vaccination campaign. One of the crucial reasons is the sceptical attitude of public towards the protection offered by the in use COVID-19 vaccines. Till date, the real-world effectiveness of the COVID vaccines used in India is not documented. We generated the evidence on the effectiveness of COVID-19 vaccines used in India through this study. We found the partial vaccination to be $52 \%$ effective in preventing a SARS-CoV-2 infection and that the two doses to be $83 \%$ effective. The COVID-19 vaccination was found to reduce the LOS and likelihood of severe disease among cases.

We found the single dose of COVID-19 vaccines to be $52.0 \%$ effective which was low compared to the efficacy of $76.0 \%$ claimed by Oxford-AstraZeneca excluding the initial 21 days postvaccination [4]. VIVALDI cohort study [25], which requited older adults ( $\geq 65$ years) of the UK as study participants, reported $68 \%$ risk reduction (at 35-48 days) of contracting SARS-CoV-2 following a single jab of Oxford-AstraZeneca vaccine. Even higher risk reduction of infection ( $74 \%$ ( 28 days onwards)) following a single standard dose of Oxford-AstraZeneca vaccine was claimed by Glampson et al. [26] which enrolled participants of all ages $(\geq 16$ years) in their study. Both these observations were higher compared to us. Higher single-dose effectiveness of Oxford-AstraZeneca vaccine has also been reported by Lopez Bernal et al. [17] $(60 \%$ (between 28 and 34 days), 73\% (post 35 days)) from the UK using a test-negative case-control design among older adults $(\geq 70$ years). There could be multiple reasons for the differences in the VE results in our study and the studies reported from the UK. The foremost reason is the age of the study participants. The chance of getting infection and having COVID-19 increases with age, so also the VE $[27,28]$. The participants in all the UK studies were comparatively older than our study except for the study reported from north-west London [26]. Lopez Bernal et al. [17] examined VE for disease prevention by contrast we checked VE for infection prevention. Generally, the VE of disease prevention is higher than the VE of infection prevention [29]. Second, unlike us, Shrotri et al. [25] and Glampson et al. [26] adopted a cohort study design for VE assessment. Moreover, ethnic variations might have played a role. Lastly, all the UK studies were done during December 2020 to March 2021 when the predominant viral strain circulating in that country was B.1.1.7 [30]. During our survey, the predominant SARS-CoV-2 variant circulating in our country was B.1.617 [31]. Viral strain variation is a known influencer of VE [29]. We found the effectiveness of $83.0 \%$ after full vaccination in India. The vaccine efficacy $(81.3 \%)$ reported by Oxford-AstraZeneca with two standard doses at $\geq 12$ weeks interval was similar to our observations [4]. The Covaxin phase III trial has reported vaccine efficacy of $77.8 \%$ in the prevention of symptomatic COVID-19 which was also in line with our observations [3]. We claim our results to be more accurate, not only due to adjustment for the potential and actual confounders, but also the inclusion of 'COVID-appropriate behaviour following vaccination' in the confounder list which others have omitted due to varied reasons. 
Table 1. Background characteristics of the study participants

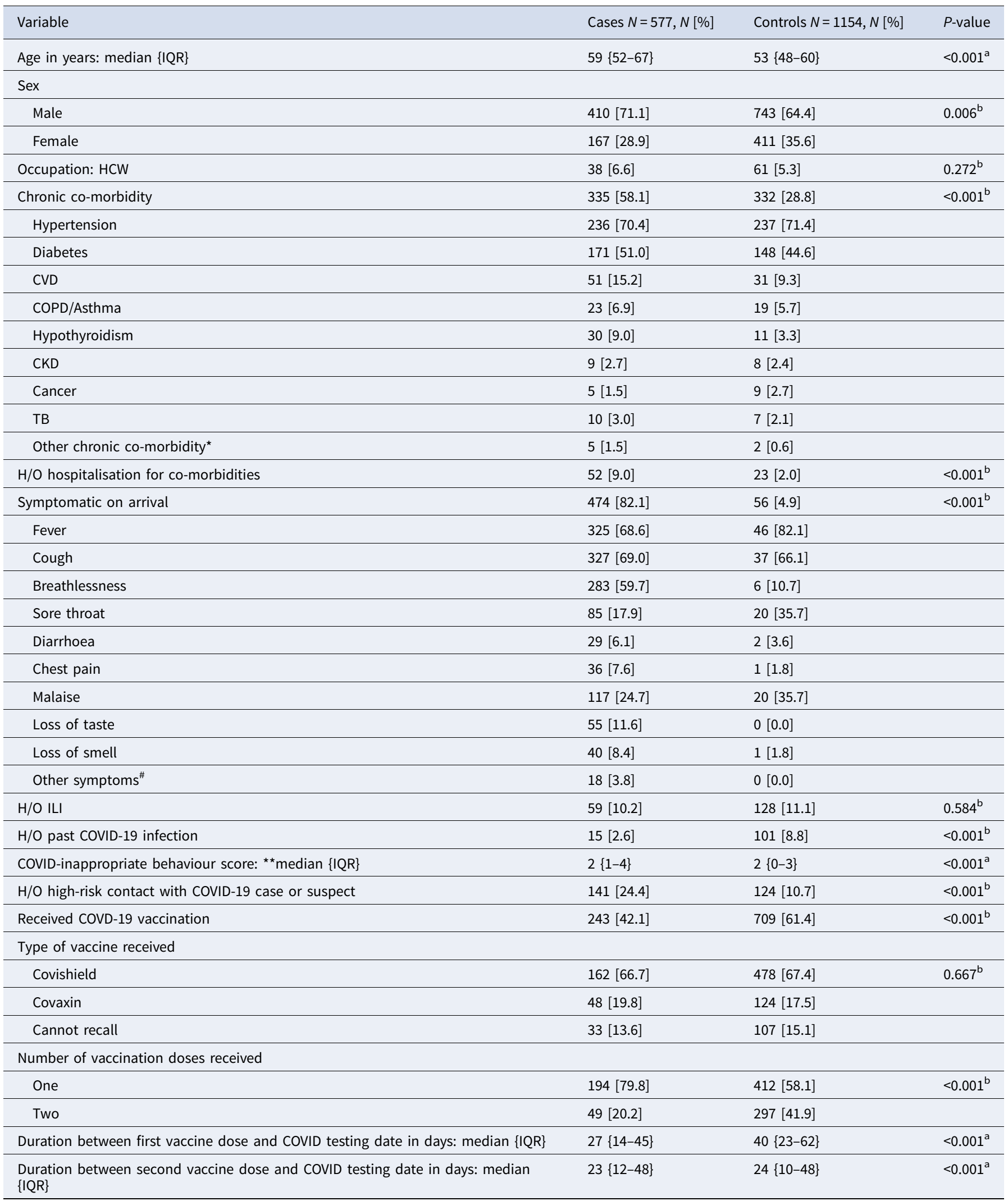

HCW, health care worker; CVD, cardiovascular disease; COPD, chronic obstructive pulmonary disease; CKD, chronic kidney disease; TB, tuberculosis; H/O, history of; COVID-19, corona virus disease-19; ILI, influenza-like illness; IQR, interquartile range; RTPCR, reverse transcriptase polymerase chain reaction.

${ }^{a}$ Independent samples Mann-Whitney $U$ test.

${ }^{\mathrm{b}} \chi^{2}$ test.

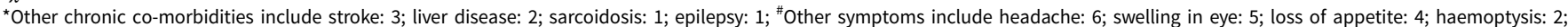
nausea/vomiting: 2 . ${ }^{\star *}$ It was calculated based on the use of mask while going outdoors, adherence to social distancing, avoidance of crowded places, handwashing before touching face in preceding 14 days of COVID testing. \{ \}: interquartile range; [ ]: column percentage. 


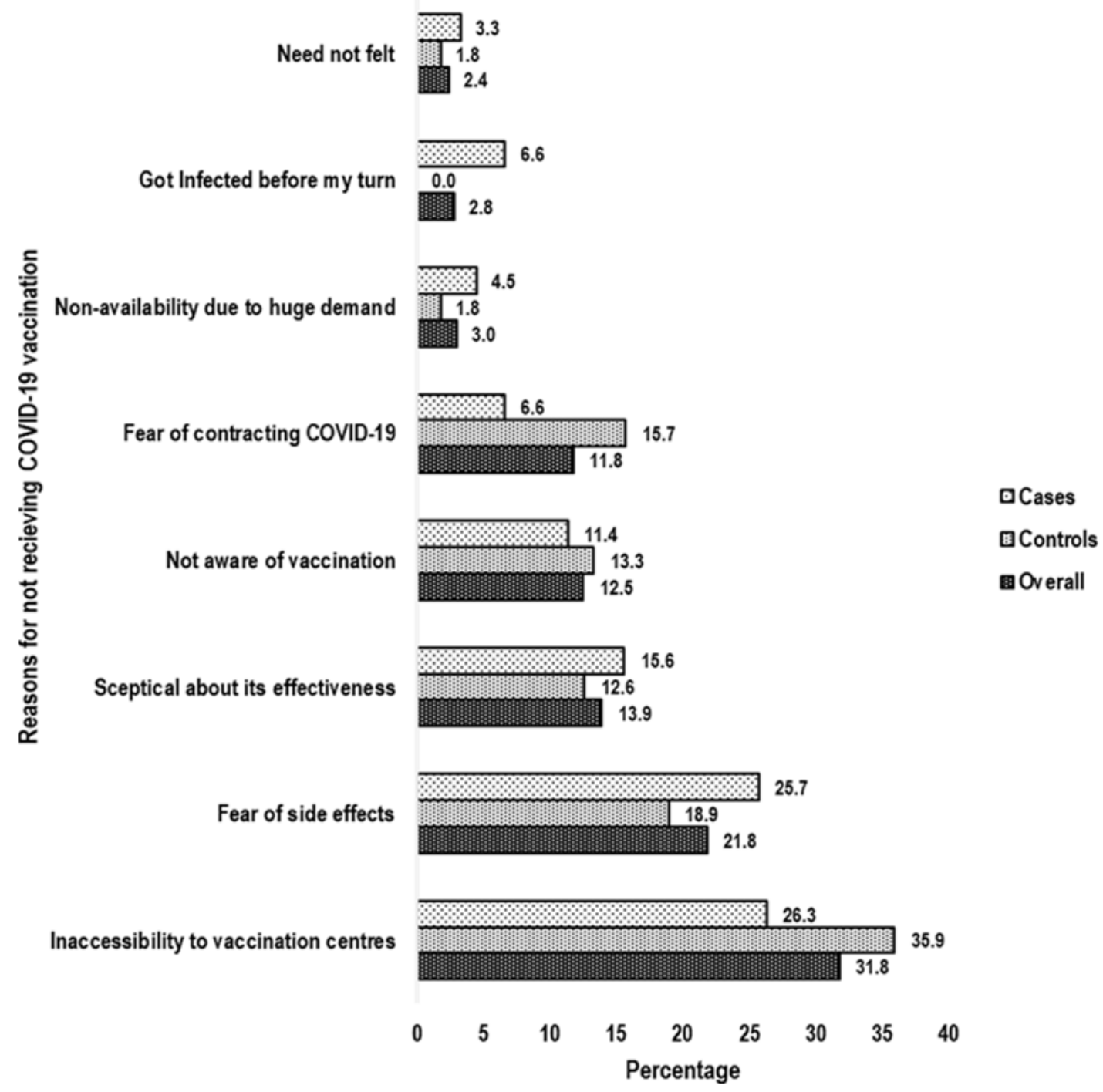

Fig. 2. Bar chart showing predominant reasons for not receiving COVID-19 vaccination. COVID-19, corona virus disease-19.

The predominant reason for not receiving vaccine against COVID-19 was reported to be the inaccessibility to the vaccination centre. Online registration through CoWIN app is mandatory to get vaccine in India. Signal cloud congestion along with the necessity for prior appointment to get the vaccine restricted the accessibility to the vaccination centre. Above that the low literacy in Bihar [19] would have contributed towards the poor understanding and operations of CoWIN App among the beneficiaries. The second and third most common reasons behind nonvaccination were the fear of side effects and sceptical about VE. The findings of this study can be used to establish the trust of common people and help improve the vaccination coverage in the fight against the COVID-19.

\section{Strengths and limitations}

This is one of the earlier evidences on the VE of COVID-19 vaccines used in India. Despite an extensive literature search, we could not come across a published piece of literature on the VE of COVID vaccines used in India. We have recruited two controls for each case which has increased the power of the study. The post-hoc power analysis revealed that the study had $100.0 \%$ and $88.8 \%$ power to elicit the differences between cases and controls in terms of full and partial vaccination, respectively. The controls recruited were RT-PCR-negative for SARS-CoV-2 ribonucleic acid (RNA). We have also adjusted for the confounders in the final analysis. Most importantly, we have adjusted for the COVID-19-appropriate behaviour in our study.

Like any other observational study, our study also has few limitations. First, we recruited cases only from one centre, AIIMS, Patna which is the highest referral institute for the management of COVID-19 in Bihar. Patients from all the corners of the state are being referred here. Thus, the cases of the present study might represent COVID-19 cases for the state of Bihar to a large extent. Second, the telephonic interview was used as a mode of data collection for home-isolated cases and controls. Inherent biases associated with the telephonic interview might be present. The telephonic interviews were made by trained resident doctors, and repeat calls following consent were made in case of any doubt over a piece of information. This might have alleviated the bias to some extent. Third, the errors associated with 
Table 2. Vaccine effectiveness in preventing COVID-19 infection

\begin{tabular}{|c|c|c|c|c|c|c|c|}
\hline \multirow[b]{2}{*}{ Variables } & \multirow{2}{*}{\begin{tabular}{l}
\multicolumn{1}{c}{ Total } \\
$N=1731$, \\
$N[\%]$
\end{tabular}} & \multicolumn{2}{|c|}{ COVID-19 infection } & \multirow[b]{2}{*}{$\begin{array}{l}\text { Unadjusted odds ratio, } \\
\text { OR }(95 \% \mathrm{Cl})\end{array}$} & \multirow[b]{2}{*}{$\begin{array}{c}\text { Unadjusted vaccine } \\
\text { effectiveness } \%(95 \% \mathrm{Cl})\end{array}$} & \multirow[b]{2}{*}{$\begin{array}{l}\text { Adjusted odds ratio, } \\
\text { OR }(95 \% \mathrm{Cl})\end{array}$} & \multirow{2}{*}{$\begin{array}{c}\text { Adjusted vaccine } \\
\text { effectiveness \% } \\
\text { (95\% Cl) }\end{array}$} \\
\hline & & $\begin{array}{l}\text { Yes, } N=577 \text {, } \\
\quad N(\%)\end{array}$ & $\begin{array}{l}\text { No, } N=1154 \\
\quad N(\%)\end{array}$ & & & & \\
\hline $\begin{array}{l}\text { Age in completed years: median } \\
\{\text { IQR }\}\end{array}$ & $\begin{array}{l}55\{50- \\
63\}\end{array}$ & $59\{52-67\}$ & $53\{48-60\}$ & $1.05(1.04-1.07)$ & - & $1.05(1.04-1.06)$ & - \\
\hline Gender: males & $\begin{array}{l}1153 \\
{[66.6]}\end{array}$ & $410(35.6)$ & $743(64.4)$ & $1.36(1.09-1.69)$ & - & $1.36(1.06-1.73)$ & - \\
\hline Occupation: HCW & $99[5.7]$ & $38(38.4)$ & $61(61.6)$ & $1.26(0.83-1.92)$ & - & $1.87(1.11-3.14)$ & - \\
\hline Chronic co-morbidity: yes & $667[38.5]$ & $335(50.2)$ & $332(49.8)$ & $3.43(2.78-4.22)$ & - & $2.51(1.97-3.19)$ & - \\
\hline $\begin{array}{l}\text { H/O hospitalisation for } \\
\text { co-morbidities: yes }\end{array}$ & $75[4.3]$ & $52(69.3)$ & $23(30.7)$ & $4.87(2.95-8.04)$ & - & $3.11(1.74-5.57)$ & - \\
\hline H/O ILI: no & $\begin{array}{l}1544 \\
{[89.2]}\end{array}$ & $518(33.5)$ & $1026(66.5)$ & $1.09(0.79-1.52)$ & - & $1.08(0.72-1.62)$ & - \\
\hline H/O past COVID-19 infection: no & $\begin{array}{l}1615 \\
{[93.3]}\end{array}$ & $562(34.8)$ & $1053(65.2)$ & $3.59(2.07-6.24)$ & - & $5.33(2.83-10.04)$ & - \\
\hline $\begin{array}{l}\text { COVID-inappropriate behaviour } \\
\text { score: }{ }^{a} \text { median }\{\mathrm{IQR}\}\end{array}$ & $2\{0-4\}$ & $2\{1-4\}$ & $2\{0-3\}$ & $1.15(1.09-1.20)$ & - & $1.12(1.05-1.18)$ & - \\
\hline $\begin{array}{l}\text { H/O high-risk contact with } \\
\text { COVID-19 case or suspect: yes }\end{array}$ & 265 [15.3] & $141(53.2)$ & $124(46.8)$ & $2.69(2.06-3.50)$ & - & $3.86(2.80-5.31)$ & - \\
\hline \multicolumn{8}{|l|}{ Vaccination status $^{\mathrm{b}}$} \\
\hline Partially vaccinated & $550[31.8]$ & $154(28.0)$ & $396(72.0)$ & $0.55(0.44-0.70)$ & $45.0(30.0-56.0)$ & $0.48(0.37-0.61)$ & $52.0(39.0-63.0)$ \\
\hline Fully vaccinated & 234 [13.5] & $33(14.1)$ & $201(85.9)$ & $0.23(0.16-0.35)$ & $77.0(65.0-84.0)$ & $0.17(0.11-0.27)$ & $83.0(73.0-89.0)$ \\
\hline
\end{tabular}

COVID-19, corona virus disease-19; IQR, interquartile range; HCW, health care worker; H/O, history of; ILI, influenza-like illness; OR, odds ratio; CI, confidence interval. Bivariate and multivariable logistic regression was done to calculate OR.

${ }^{\text {at }}$ was calculated based on the use of mask while going outdoors, adherence to social distancing, avoidance of crowded places, handwashing before touching face in preceding 14 days of COVID testing.

${ }^{b}$ The definitions used for ascertaining COVID-19 vaccination status were as follows, unvaccinated: at the time of testing for SARS-CoV-2, one has not received jab or was within 14 days of first jab; partially vaccinated: did the testing for SARS-CoV-2, $\geq 14$ days of first jab or before 14 days of second jab; fully vaccinated: did the testing for SARS-CoV-2, $\geq 14$ days after second jab. \{ \}: interquartile range; [ ]: column percentage; (): row percentage. The vaccine effectiveness of the Covishield and Covaxin subgroups is being reported in Supplementary Tables S1 and S2 respectively. 


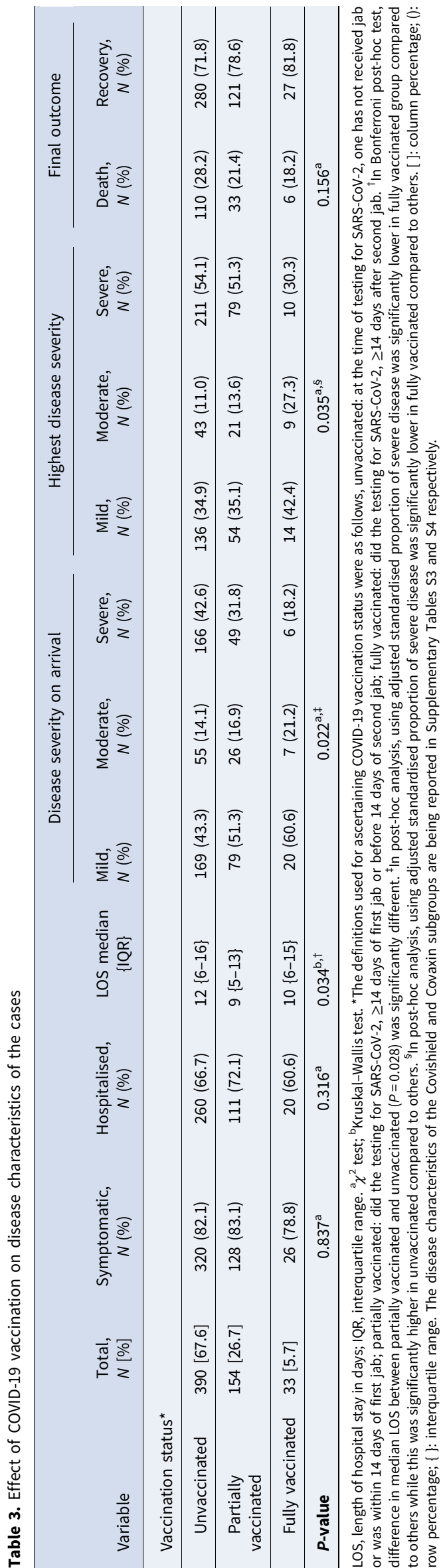

self-reporting of some information. We tried to reduce these errors by developing rapport with the participant and allowing sufficient time to respond. We also requested them to confirm the vaccination details by cross-checking with the CoWIN app or the message received following vaccination. Fourth, the misclassification due to the inherent properties of the test used for the investigation of SARS-CoV-2. The RT-PCR-based testing is only $60-70 \%$ sensitive, although specificity is claimed to be more than $90 \%$. Lastly, we did not have a sufficient sample size to do sub-group analysis for Covishield and Covaxin groups. However, the analysis and findings with the samples at our hand are provided in Supplementary Tables.

\section{Conclusions}

COVID-19 vaccination was found to be effective in infection prevention. One out of two and four out of five individuals were found to be protected against SARS-CoV-2 infection following partial and full vaccination, respectively. The vaccinated individuals had lesser LOS compared to unvaccinated ones. Additionally, the fully vaccinated individuals were less likely to develop severe disease. We recommend studies with a larger sample size to elicit the VE of individual COVID-19 vaccines.

Supplementary material. The supplementary material for this article can be found at https://doi.org/10.1017/S0950268821002247

Acknowledgements. We place in records the amazing efforts from the junior residents of the Department of Community and Family Medicine, AIIMS, Patna for the data collection. We also acknowledge the help extended by the Department of Medical Records, AIIMS Patna for the provision of access to the records of the admitted COVID-19 patients to assess the highest severity and final outcome.

Author contributions. Conceptualisation: CS, PKS, BNN, SP, BB; data curation: $\mathrm{BB}, \mathrm{MV}, \mathrm{BKP}$; formal analysis: $\mathrm{BB}, \mathrm{BNN}$; funding acquisition: $\mathrm{NA}$; investigation: CS, PKS, BNN, SP, BB, BKP, MV; methodology: CS, BNN, SP, $\mathrm{BB}$; project administration: $\mathrm{BB}, \mathrm{BNN}, \mathrm{MV}, \mathrm{BKP}$; resources: $\mathrm{CS}, \mathrm{SP}, \mathrm{BKP}$, $\mathrm{BNN}$; software: $\mathrm{BB}, \mathrm{BNN}$; supervision: $\mathrm{CS}, \mathrm{BNN}, \mathrm{SP}, \mathrm{BB}, \mathrm{MV}$; validation: $\mathrm{BNN}, \mathrm{PKS}, \mathrm{BB}$; visualisation: $\mathrm{CS}, \mathrm{BNN}, \mathrm{SP}, \mathrm{BB}$; writing - original draft: $\mathrm{BB}$, BNN; writing - review and editing: CS, PKS, BNN, BB, SP, BKP, MV.

Financial support. This research received no specific grant from any funding agency in the public, commercial, or not-for-profit sectors.

Conflict of interest. None.

Informed consent. Informed written consent of cases and informed verbal consent of controls were sought before study tool administration.

Institutional review board approval. Ethical clearance was obtained from the Institutional Ethics Committee of AIIMS-Patna (Ref. No. - AIIMS/Pat/ IEC/2021/706).

Ethical compliance with human/animal study. This study was conducted in compliance with the ethical standards of the responsible institution on human subjects as well as with the Helsinki Declaration.

Data availability. All data generated or analysed during this study are included in this published article (and its Supplementary information files).

\section{References}

1. Vaccines and immunization. Available at https://www.who.int/healthtopics/vaccines-and-immunization (Accessed 11 July 2021).

2. India: WHO Coronavirus Disease (COVID-19) Dashboard with Vaccination Data. Available at https://covid19.who.int (Accessed 21 July 2021). 
3. Ella R, et al. (2021) Efficacy, safety, and lot to lot immunogenicity of an inactivated SARS-CoV-2 vaccine (BBV152): a, double-blind, randomised, controlled phase 3 trial. Infectious Diseases (except HIV/AIDS), 2021 Jul. Available at http://medrxiv.org/lookup/doi/10.1101/2021.06.30. 21259439 (Accessed 11 July 2021).

4. Voysey M, et al. (2021) Single-dose administration and the influence of the timing of the booster dose on immunogenicity and efficacy of ChAdOx1 nCoV-19 (AZD1222) vaccine: a pooled analysis of four randomised trials. Lancet (London, England) 397, 881-891.

5. India rolls out the world's largest COVID-19 vaccination drive. Available at https://www.who.int/india/news/feature-stories/detail/india-rollsout-the-world-s-largest-covid-19-vaccination-drive (Accessed 11 July 2021).

6. CoWIN Dashboard. Available at https://dashboard.cowin.gov.in/ (Accessed 12 July 2021).

7. Samantaray A, et al. (2021) COVID-19: A game of drugs, vaccines, hope and... death!. Indian Journal of Anaesthesia 65, 434-438.

8. Voysey M, et al. (2021) Safety and efficacy of the ChAdOx1 nCoV-19 vaccine (AZD1222) against SARS-CoV-2: an interim analysis of four randomised controlled trials in Brazil, South Africa, and the UK. The Lancet 397, 99-111.

9. Patel MM, Jackson ML and Ferdinands J (2020) Postlicensure Evaluation of COVID-19 Vaccines. JAMA 324, 1939-1940.

10. Evaluation of COVID-19 vaccine effectiveness. Available at https://www. who.int/publications-detail-redirect/WHO-2019-nCoV-vaccine_effectivenessmeasurement-2021.1 (Accessed 21 April 2021).

11. Sallam M (2021) COVID-19 vaccine hesitancy worldwide: a concise systematic review of vaccine acceptance rates. Vaccines 9,160

12. Government of India (2020) COVID-19 Statewise Vaccine. Available at https://mygov.in/covid-19/ (Accessed 18 April 2021).

13. Araki K, et al. (2019) Case-control study of rotavirus vaccine effectiveness compared to test-negative controls or hospital controls. Journal of Epidemiology 29, 282-287.

14. Franke MF, et al. (2018) Long-term effectiveness of one and two doses of a killed, bivalent, whole-cell oral cholera vaccine in Haiti: an extended case-control study. The Lancet. Global Health 6, e1028-e1035.

15. Choi WS, et al. (2013) Case-control study of the effectiveness of the 20102011 seasonal influenza vaccine for prevention of laboratory-confirmed influenza virus infection in the Korean adult population. Clinical and vaccine immunology: CVI 20, 877-881.

16. Pilishvili T, et al. (2021) Interim estimates of vaccine effectiveness of Pfizer-BioNTech and Moderna COVID-19 vaccines among health care personnel - 33 U.S. sites, January-March 2021. MMWR. Morbidity and Mortality Weekly Report 70, 753-758.

17. Lopez Bernal J, et al. (2021) Effectiveness of the Pfizer-BioNTech and Oxford-AstraZeneca vaccines on covid-19 related symptoms, hospital admissions, and mortality in older adults in England: test negative case-control study. BMJ 373, n1088.

18. Hyams C, et al. (2021) Effectiveness of BNT162b2 and ChAdOx1 nCoV-19 COVID-19 vaccination at preventing hospitalisations in people aged at least 80 years: a test-negative, case-control study. The Lancet. Infectious Diseases S1473-3099, 00330-3. Online ahead of print.

19. Bihar Population Sex Ratio in Bihar Literacy rate data 2011-2021. Available at https://www.census2011.co.in/census/state/bihar.html (Accessed 18 April 2021).

20. OpenEpi - Sample Size for Unmatched Case-Control Studies. Available at https://www.openepi.com/SampleSize/SSCC.htm (Accessed 18 April 2021).

21. OpenEpi - Toolkit Shell for Developing New Applications. https://www. openepi.com/Power/PowerCC.htm (Accessed 11 July 2021).

22. Comprehensive Guidelines for Management of COVID-19 patients (2021) Directorate General of Health Services, Ministry of Health and Family Welfare, Government of India.

23. Standard Operating Procedure for \& Handbook for COVID-19 Management (2021) All India Institute of Medical Sciences, Patna.

24. Free and easy-to-use mobile data-gathering platform. Available at https://five.epicollect.net/\# (Accessed 21 July 2021).

25. Shrotri M, et al. (2021) Vaccine effectiveness of the first dose of ChAdOx1 nCoV-19 and BNT162b2 against SARS-CoV-2 infection in residents of long-term care facilities in England (VIVALDI): a prospective cohort study. The Lancet. Infectious Diseases S1473-3099, 00289-9. Online ahead of print.

26. Glampson B, et al. (2021) Assessing COVID-19 vaccine uptake and effectiveness through the North West London Vaccination Program: retrospective cohort study. JMIR Public Health and Surveillance 7, e30010. doi: $10.2196 / 30010$.

27. Crimmins EM (2020) Age-related vulnerability to coronavirus disease 2019 (COVID-19): biological, contextual, and policy-related factors. The Public Policy and Aging Report 30, 142-146.

28. Mueller AL, McNamara MS and Sinclair DA (2020) Why does COVID-19 disproportionately affect older people? Aging 12, 9959-9981.

29. Vaccine efficacy, effectiveness and protection. Available at https://www. who.int/news-room/feature-stories/detail/vaccine-efficacy-effectiveness-andprotection (Accessed 22 July 2021).

30. Nyberg T, et al. (2021) Risk of hospital admission for patients with SARS-CoV-2 variant B.1.1.7: cohort analysis. BMJ 373, n1412.

31. B.1.617 variant dominant strain in India: Harsh Vardhan India News Times of India. Available at https://timesofindia.indiatimes.com/india/b-1617-variant-dominant-covid-mutant-strain-in-india-accounts-for-55-of-totalvariants-of-concern-detected/articleshow/82926650.cms (Accessed 22 July 2021). 